\title{
PATTERN OF CANDIDA URINARY TRACT INFECTIONS AMONG CANCER PATIENTS IN SOUTH EGYPT CANCER INSTITUTE
}

\author{
Nabila M Rashwan ${ }^{1}$, Abdel-Khalk A Mohamed ${ }^{1}$, Salwa Saif El-Deen ${ }^{1}$, Entsar H Ahmed ${ }^{1}$ and \\ Shaymaa Abdalla Ismail ${ }^{2}$
}

${ }^{1}$ Department of Microbiology and Immunology, Faculty of Medicine, Assuit University

${ }^{2}$ South Egypt Cancer Institute, Assuit University

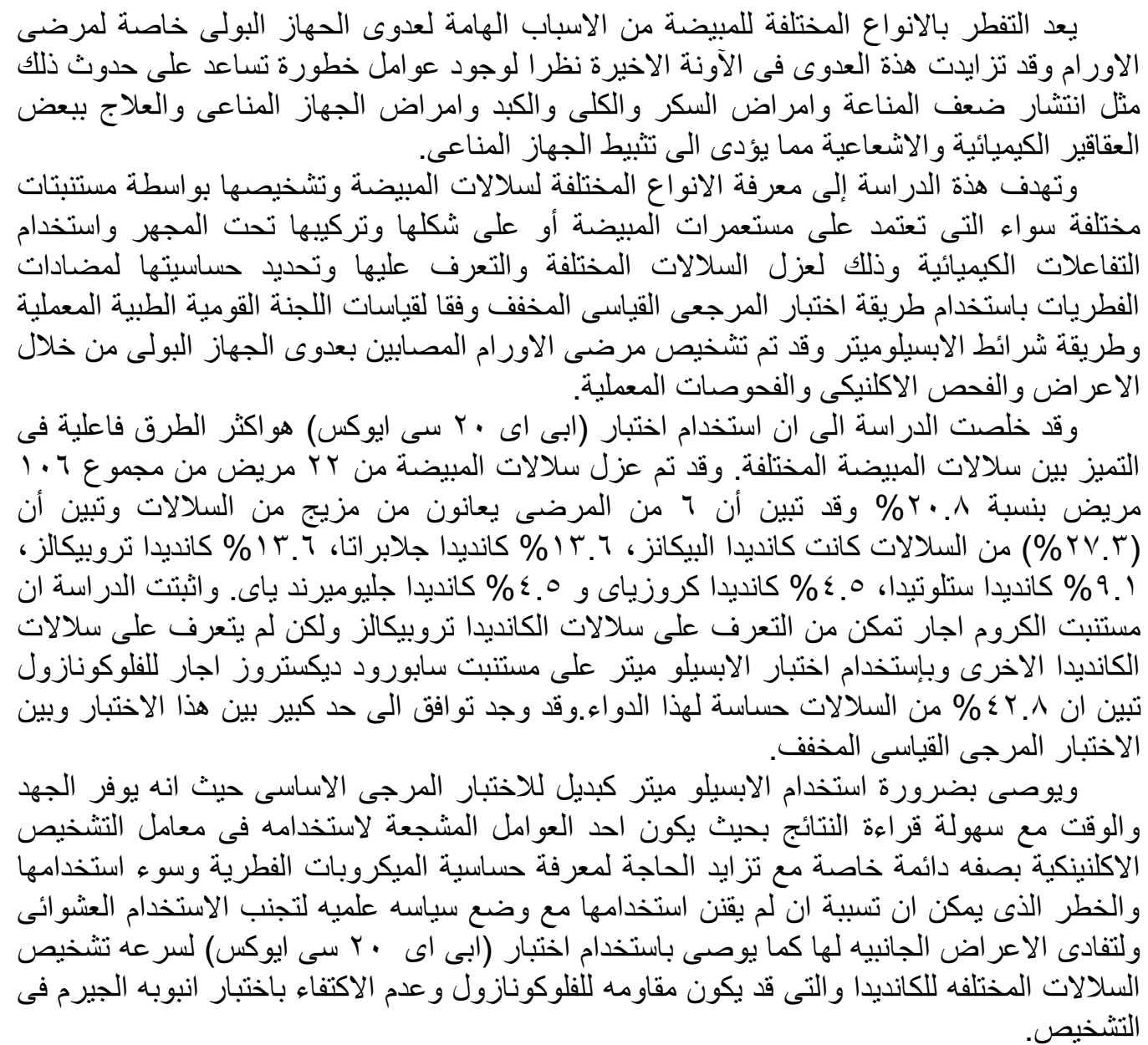

The last decade has seen the sustained medical importance of opportunistic infections due to different Candida species mainly because of the worldwide increasing in the number of immunocompromised patients, who are highly susceptible to opportunistic infections. Urine samples were collected from 106 cancer patients in South Egypt Cancer Institute (SECI) that were cultured on Sabouraud dextrose agar media for isolation of Candida species. After Gram staining subculture was done on Hicrome Candida Differential Agar media. Results of the previous media were compared with those obtained with API 20C AUX yeast identification kits. The study revealed an overall isolation rate of Candida species among urinary tract infections was $20.8 \%$ (22/106). Single type of Candida species was isolated from cancer patients with candiduria 16/22 (72.7\%) while six Patients had mixed species. Candida albicans was the most frequent species isolated responsible for fungal urinary tract infections 27.3\% (6/22). NonCandida albicans species including Candida tropicalis (13.6\%), Candida glabrata (13.6\%), Candida stellatoides (9.1\%), Candida krusei (4.5\%) and Candida guilliermondii (4.5\%) were also isolated. Candida albicans, Candida stellatoides and Candida guilliermondii could not be 
identified on chrom agar as all the isolates gave similar green colonies. Also Candida glabrata and Candida krusei could not be identified on chrom agar as they gave similar white colonies. Chrom agar identifies all Candida tropicalis as the isolates gave the typical pattern of purple to blue colonies. Candida albicans identified on Czapek Dox Agar media as they produced chlamydospores. The results of API 20C AUX were in 100\% agreement with the results of Chrom agar in identification of Candida tropicalis. E- test on (SDA) was found to be an accurate method for antifungal susceptibility as it was compared with the reference broth microdilution method recommended by National Committee for Clinical Laboratory Standards (NCCLs). For fluconazole the E-test demonstrated $94.1 \%$ agreement for all candida species.

\section{INTRODUCTION}

Identification of yeasts isolated from clinical specimens is often problematic for diagnostic laboratories, but it has become increasingly necessary. Greater numbers of immunosuppressed patients, a widening range of recognized pathogens and the discovery of resistance to antifungal drugs mean that the common practice of identification or exclusion of Candida species is no longer adequate ${ }^{1 \& 2}$. Incidence of Candidiasis causing urinary tract infection continues to rise in proportion to a growing number of patients at risk for infection with Candida albicans and recently with innately azole-resistant non-albicans Candida species $^{3 \& 4}$. This category of patients had increased as a result of more intensive regimens of cancer therapy, diabetes, complications of abdominal or cardiothoracic surgery, organ transplantations, burns and trauma ${ }^{5 \& 6}$. Common risk factors include prolonged broad spectrum antibiotic therapy, urinary catheterization, invasive devices and prolonged hospital stays $^{7 \& 8}$. Identification of Candida species can be done using commercially available chromogenic differential media; Chrom agar ${ }^{9}$. All the Candida isolates are also identified using API 20C AUX ${ }^{10 \& 11}$. Fluconazole antifungal is the standard treatment for Candidiasis; however, due to the commonly occurring, intrinsic or acquired resistance of Candida glabrata and Candida krusei to fluconazole, infections by these strains may necessitate alternative treatment with amphotericin B or itraconazole $^{12}$. E-test is a stable agar gradient strips that consist of drug concentration scale. It determines the MIC of different antifungal agents in a rapid and simple method ${ }^{13 \& 14}$. The aim of this study was to assess the role of different Candida species in urinary tract infections among cancer patients in SECI. Also, identification of different species using differential media as well as determination of antifungal susceptibility testing to fluconazole using E-test and broth microdilution methods.

\section{PATIENTS, MATERIAL AND METHODS}

This study included 106 patients with cancer presented to South Egypt Cancer Institute suffering from urinary tract infection. They were 74 males and 32 females. Their ages ranged from 5 to 86 years old. They were divided into 2 groups:

Group I: patients suffering from genitourinary tract cancer (83 patients).

Group II: Patients suffering from other type of cancer (23 patients).

All patients were subjected to the following: Detailed history of any previous urologic pathology, Operation, use of antibiotics, cancer chemotherapy, use of corticosteroids or immunosuppressive drugs, impaired kidney function. Patients are subjected to thorough clinical examination, complete routine microscopic urine examination.

Samples collection: Urine specimens were collected from all patients clinically diagnosed to have urinary tract infection by midstream or through catheter under aseptic technique in sterile containers ${ }^{15}$. The specimens were examined and cultured without delay (within 1 hour of collection and kept in ice box until processing). Urine sample was concentrated by centrifugation then a smear was prepared from the sediment and gram stained then examined microscopically. A loopful was taken from the sediment and cultured on Sabouraud dextrose agar, and then incubated aerobically for 24 to 48 hours at $37^{\circ} \mathrm{C}$. Colonies identified as Candida by Gram-stain were subjected to germ tube test ${ }^{16 \& 17}$. Isolated colony of Candida species was subcultured on CHROM agar then 
incubated aerobically at $30^{\circ} \mathrm{C}$. The growth of Candida species was inspected at 24, 48 and 72 hours then interpreted according to the chromogenic scheme of the media ${ }^{1 \& 9}$.

Subculture on czapek Dox Agar media: Czapek Dox Agar (Modified) was a medium containing sodium nitrate as the sole source of nitrogen; it was one of the most useful solid media for the general cultivation of fungi. The medium was a highly satisfactory substrate for chlamydospore production by Candida albicans ${ }^{18}$.

\section{Important biochemical reactions including carbohydrate assimilation test: All the Candida isolates were also identified using API 20C AUX (Himedia,Mombai, India) using the manual instructions and identified through specific number code ${ }^{10 \& 11}$.}

\section{Antifungal susceptibility test: Minimal inhibitory concentration (MIC) using microdilution broth method is used according} to NCCLs M27-T guideline (National Committee for Clinical Laboratory standards (NCCLs) 2002) for antifungal susceptibility testing to fluconazole. Serial dilutions of antifungal were prepared. The final concentrations of fluconazole (Pfizer, Inc., New York, NY) ranged from 0.25 to 128 $\mu \mathrm{g} / \mathrm{ml}^{19}$. Five colonies on the SAB plate were taken with a sterile loop and suspended in $0.85 \%$ saline in the spectrophotometer tube that was Vortexed for $10 \mathrm{sec}$ then the $\%$ transmission (T) was read. The reading was between $85-90 \% \mathrm{~T}$. More saline was added for adjustment to raise the $\% \mathrm{~T}$ or more cell suspension was added to decrease the $\% \mathrm{~T}$. This resulted a cell count of about 1 to $5 \times 10^{6}$ $\mathrm{CFU} / \mathrm{ml}$. This stock was diluted 1000 -fold for preparation of the working suspention. This was achieved by adding $0.1 \mathrm{ml}$ of stock to 4.9 $\mathrm{ml}$ of RPMI medium (50-fold dilution) followed by adding $0.5 \mathrm{ml}$ of this diluted stock to $9.5 \mathrm{ml}$ of RPMI medium (20-fold dilution). This resulted in a suspension of twice the final inoculum concentration of $2.5 \times 10^{3} \mathrm{CFU} / \mathrm{ml}$. $100 \mu 1$ of the working suspension was added in RPMI medium to each of the wells in a row except column 12 (inoculum-free control).

Epsilometry test (E-Test) strips (Himedia, Mumbai, India): It gives a quantitative accurate measure of the MIC for testing the invitro susceptibilities of clinical isolates of Candida species to antifungal agents. The strips consisted of drug concentrations ranging from $0.016 \mu \mathrm{g} / \mathrm{ml}$ to $256 \mu \mathrm{g} / \mathrm{ml}$ for fluconazole. SDA media plates are used for inoculation using a swab dipped in a cell suspension adjusted optically $(0.08-0.13$ optical density turbid suspension at $\mathrm{nm}$ yields $10^{5}-10^{6}$ cells $/ \mathrm{ml}$ ). The plates were incubated at $35^{\circ} \mathrm{C}$ and read after 18-24 hours. The determination of the MICs of antifungal agents is based on reading the lowest concentration at which the border of the elliptical inhibition zone intercepted the scale on the strip. Azoles being fungistatic, so appearance of microcolonies throughout the discernible inhibition ellipse is ignored ${ }^{13 \& 20}$.

\section{Statistical analysis}

Data were represented in tables using positivity percent. Chi-square $(\mathrm{X})^{2}$ for analysis of categorically variables and correlation tests. The mean and standard deviation (SD) were used for numerical data for description. Statistical analysis was performed using the Statistical Package for Social Sciences (SPSS version 16).

\section{RESULTS AND DISCUSSION}

\section{Results}

The present study revealed an overall isolation rate of $20.8 \%(22 / 106)$ of candida species. Incidence of candida species in patients with genitourinary tract cancer was $16.9 \%$ and in other type of cancer was $34.8 \%$. They were 74 Males and 32 females. Their ages ranged from 5 to 86 years with mean age and SD of $57.03 \pm 15.1$ years. Females were found to be more prone to urinary Candidiasis $34.4 \%$ than males $14.9 \%$ with statistical significant difference using Chi square test $(\mathrm{p}=$ 0.04). Predisposing factors for urinary Candidiasis revealed that prolonged use of broad spectrum antibiotics $(\mathrm{p}=0.02)$ was the most prevalent factor being in $45.5 \%$ (10/22), chemotherapy for the treatment of cancer $(\mathrm{p}=0.04)$ being in $40.9 \%(9 / 22)$ and abdominal operation $(\mathrm{p}=0.03)$ being in $13.6 \%(3 / 22)$ of infected patients.

The frequency distribution of Candida species is shown in figure 1. Single type of Candida species was isolated from cancer 
patients with candiduria 16/22 (72.7\%). six Patients had two different types of candida species, four patients had Candida albicans in addition to Candida tropicalis or Candida parapsilosis. The remaining two patients had Candida stellatoides with Candida glabrata.The sole pathogenic organism was Candida albicans in $6 / 22(27.3 \%)$, Candida glabrata in $3 / 22$ (13.6\%), Candida tropicalis in $3 / 22(13.6 \%)$, Candida stellatoides in $2 / 22(9.1 \%)$, Candida krusei in $1 / 22(4.55 \%)$ and Candida guilliermondii in $1 / 22(4.55 \%)$ of the patients. Candida albicans was isolated in $70 \%$ of patients with Genitourinary Tract cancer in compared to $30 \%$ in other type of cancer. While Non-Candida albicans was isolated in $61.1 \%$ of patients with Genitourinary Tract cancer in compared to $38.9 \%$ in other type of cancer as shown in table 1.

Using germ tube test method all Candida albicans and Candida stellatoides were positive while all Candida glabrata, Candida krusei and Candida guilliermondii isolated were negative. Germ tube test was positive in 2 isolates of Candida tropicalis as diagnosed by Chrom agar and API 20 C AUX. Candida albicans, Candida stellatoides and Candida guilliermondii could not be identified on chrom agar as all the isolates gave similar green colonies. Also
Candida glabrata and Candida krusei could not be identified on chrom agar as they gave similar white colonies. Chrom agar identifies all Candida tropicalis as the isolates gave the typical pattern of purple to blue colonies as shown in figure 2 .

Candida albicans can be identified on Czapek Dox Agar media as they produced chlamydospores. The results of API 20C AUX were in $100 \%$ agreement with the results of Chrom agar in identification of Candida tropicalis. Susceptibility of isolated Candida species to fluconazole using E-test strips on SDA shown in figure 3 showed that $42.86 \%$ $(12 / 28)$ of the isolates were susceptible as shown in table 2. While 4 isolates of Candida albicans and Candida glabrata were reported as S-DD (susceptible dose dependent). The MIC of the clinical isolates of different candida species determined by E-test method showed comparable result to the NCCLs broth microdilution method for fluconazole $(94.1 \%$ agreement) as shown in table 3 . Since all the results of E-test method was in agreement with NCCLs broth microdilution method for all candida species except one of Candida albicans strains was S-DD in E-test method while it was resistant in NCCLs broth microdilution method.

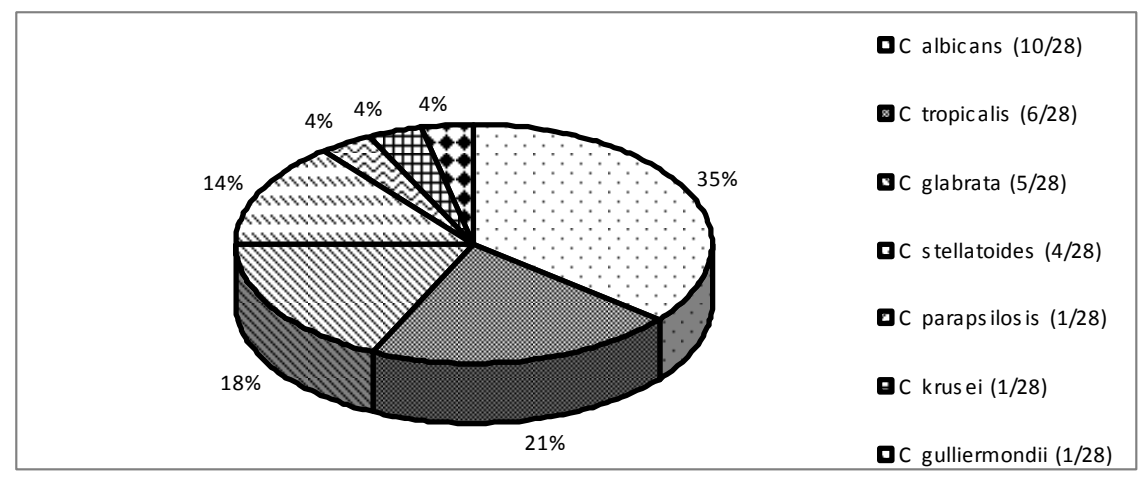

Fig. 1: Frequency distribution of candida species isolated from 22 patients with candiduria.

Table 1: Candida isolates from genitourinary tract and other type of cancer patients.

\begin{tabular}{|l|c|c|c|c||}
\hline \multirow{2}{*}{ Candida species } & \multicolumn{2}{|c|}{$\begin{array}{c}\text { Candida } \\
\text { albicans }\end{array}$} & \multicolumn{2}{c|}{$\begin{array}{c}\text { Non-Candida } \\
\text { albicans }\end{array}$} \\
\cline { 2 - 5 } & No. & $\%$ & No. & $\%$ \\
\hline Genitourinary Tract & 7 & 70 & 11 & 61.1 \\
\hline Other & 3 & 30 & 7 & 38.9 \\
\hline Total & 10 & 100 & 18 & 100 \\
\hline
\end{tabular}



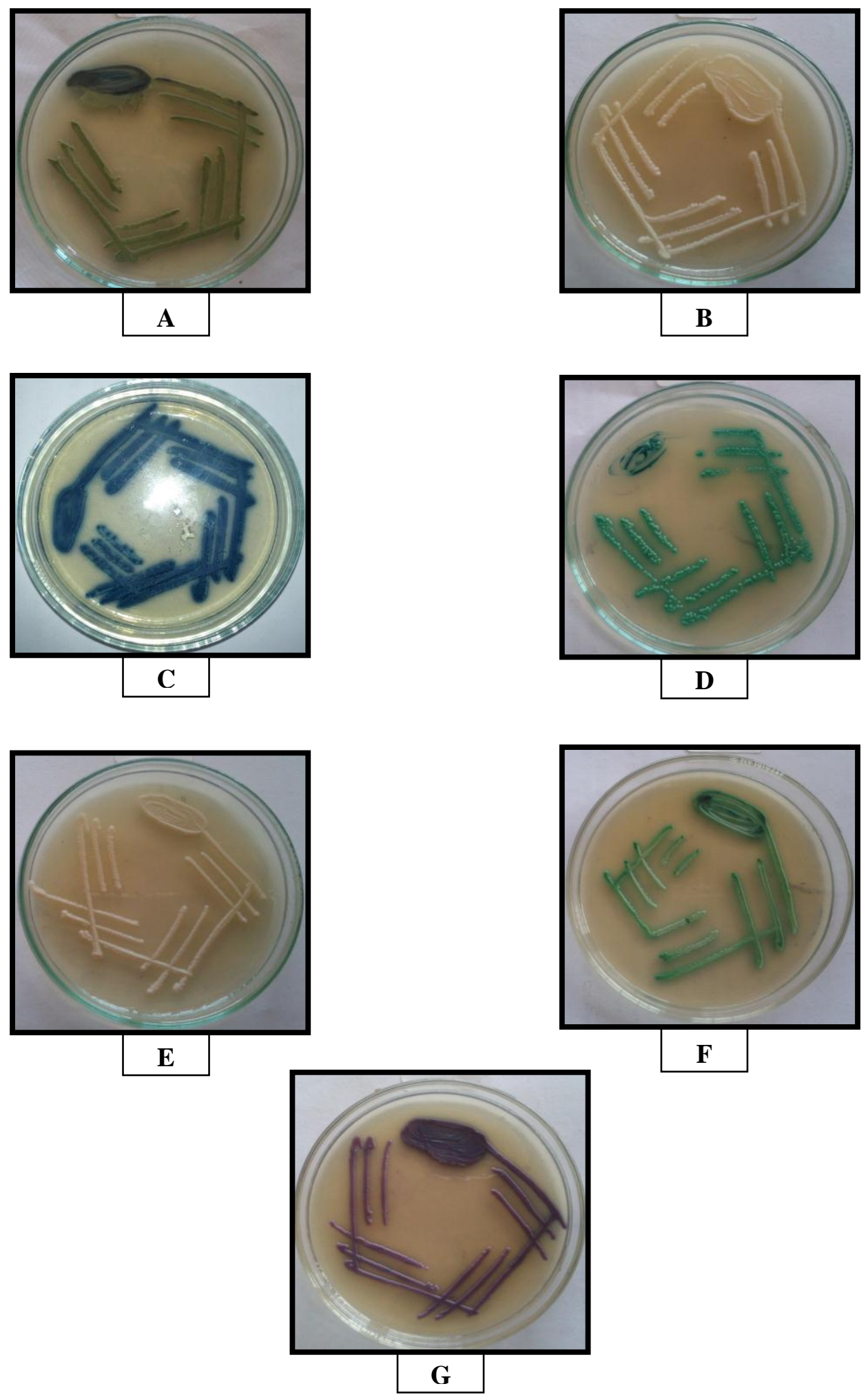

Fig. 2: Appearance of Candida species colonies on Chrom agar; A) Candida albicans.
B) Candida glabrata
C) Candida tropicalis
D) Candida stellatoides
E) Candida krusei
F) Candida guilliermondii
G) Candida parapsilosis 

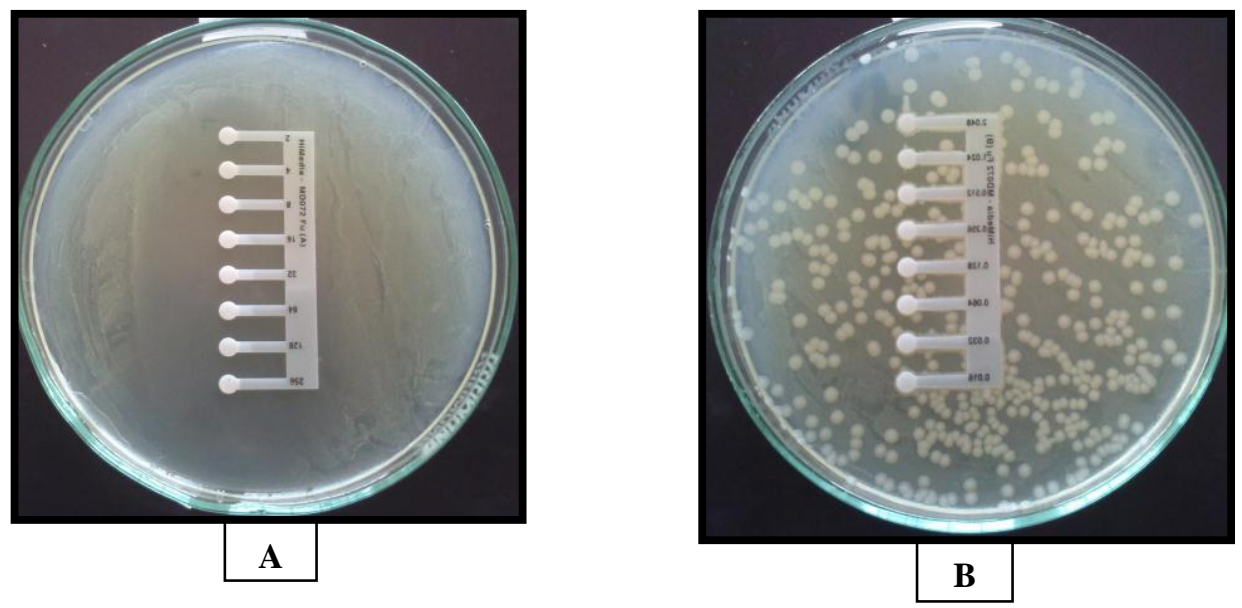

Fig. 3: E-test of fluconazole.

$\mathrm{A}=$ Range of fluconazole from 256-2, B= Range of fluconazole from 2.048-0.016.

Table 2: Susceptibility pattern to fluconazole using E-test.

\begin{tabular}{|c|c|c|c|c|}
\hline \multirow{2}{*}{ Candida species } & \multicolumn{3}{|c|}{ Sensitivity } & \multirow{2}{*}{ Total } \\
\hline & $S$ & S-DD* & $\mathrm{R}$ & \\
\hline \multirow{2}{*}{ Candida albicans } & 3 & 2 & 5 & 10 \\
\hline & $30.00 \%$ & $20.00 \%$ & $50.00 \%$ & $35.71 \%$ \\
\hline \multirow{2}{*}{ Candida tropicalis } & 3 & 0 & 3 & 6 \\
\hline & $50.00 \%$ & $0.00 \%$ & $50.00 \%$ & $21.43 \%$ \\
\hline \multirow{2}{*}{ Candida glabrata } & 2 & 2 & 1 & 5 \\
\hline & $40.00 \%$ & $40.00 \%$ & $20.00 \%$ & $17.86 \%$ \\
\hline \multirow{2}{*}{ Candida stellatoides } & 2 & 0 & 2 & 4 \\
\hline & $50.00 \%$ & $0.00 \%$ & $50.00 \%$ & $14.29 \%$ \\
\hline \multirow{2}{*}{ Candida parapsilosis } & 1 & 0 & 0 & 1 \\
\hline & $100 \%$ & $0.00 \%$ & $0.00 \%$ & $3.57 \%$ \\
\hline \multirow{2}{*}{ Candida krusei } & 1 & 0 & 0 & 1 \\
\hline & $100 \%$ & $0.00 \%$ & $0.00 \%$ & $3.57 \%$ \\
\hline \multirow{2}{*}{ Candida guilliermomdii } & 0 & 0 & 1 & 1 \\
\hline & $0.00 \%$ & $0.00 \%$ & $100 \%$ & $3.57 \%$ \\
\hline \multirow{2}{*}{ Total } & 12 & 4 & 12 & 28 \\
\hline & $42.86 \%$ & $14.28 \%$ & $42.86 \%$ & $100.00 \%$ \\
\hline
\end{tabular}

*S-DD $=$ susceptible dose dependent

Table 3: Agreement between E-test and microdilution broth method of fluconazole.

\begin{tabular}{|c|c|c|c|}
\hline Method & Fluconazole & Sensitivity & Specificity \\
\hline$* \mathrm{E} / \mathrm{BM}$ & $\begin{array}{c}27 / 28 \\
(94.1 \%)\end{array}$ & $100 \%$ & $92.3 \%$ \\
\hline
\end{tabular}

$* \mathrm{E}=\mathrm{E}$-test and $\mathrm{BM}=$ Broth Microdilution 


\section{Discussion}

Candiduria indicates the presence of Candida species in the urine ${ }^{21}$. The incidence of candiduria is estimated to be $6.5-20 \%$ among hospitalized patients ${ }^{22}$. Pakshir and colleagues reported a frequency of $28.7 \%{ }^{23}$. Badawi et $a l^{24}$, reported that isolation rate of $25 \%$ of the UTIs cases. The results of this study (isolation rate of 20.8\%) were closely nearer to Febre and colleagues $^{25}$ who reported isolation rate of $21.4 \%$. Cancer patients are the most exposed subjects to such clinical complication and Candida is one of the five most frequently isolated microbes from blood cultures. Indeed, Candida species are by far the most common cause of fungal infections and they produce infections that range from non-life threatening mucocutaneous illnesses to invasive processes that may eventually involve any body organ ${ }^{26}$. Common risk factors for candiduria are antimicrobial therapy, female gender, urinary tract abnormality, diabetes, presence of Foley catheter, older age, cancer immunosuppressive therapy and abdominal surgery ${ }^{27}$. Development of urinary infections is more common in females due to anatomical and functional reasons ${ }^{28}$. In this study candiduria was observed in $34.4 \%$ females compared to $14.9 \%$ males $(p=0.04)$. These results are in accordance with the finding of Febre et $a l^{25}$, who reported that isolation rate of $30.8 \%$ and $11.4 \%$ were detected in females and males, respectively. This is attributed to the shorter urethra of females and its proximity to the anus and vagina $^{15}$. Advanced age is known to be another risk factor in the development of candiduria. Sobel and colleagues reported that the mean age of cases was $70.2 \pm 1.2$ years $^{29}, 65$ years in the study of Febre and colleagues ${ }^{25}, 67$ years in the study of Ang and colleagues ${ }^{23}$ and 75 years in the study of Jacobs and colleagues ${ }^{30}$. Also, Harris et $a l^{31}$, reported the mean age for Candida glabrata and Candida albicans infections as 66 years. Despite these studies, which suggest advanced age as a risk factor, Kobayashi et $a l .{ }^{28}$ found the mean age of their study population with Candiduria to be 48 \pm 19.8 years. The mean age in this study was $57.03 \pm 15.1$ years. In general, hospitals particularly university hospitals use widely broad spectrum antibiotics ${ }^{32}$. Kauffman and colleagues $^{27}$ determined the presence of antibiotic use in $90 \%$ of 861 patients. This condition is determined to be the most important cause of the increase in the prevalence of candidal infections ${ }^{28}$.Various mechanisms are proposed in order to explain the relationship between the use of antibiotics and candiduria. It was shown that antibiotics impaired phagocytic activity and antibody synthesis and consequently decreased the resistance of the host against candida invasion $^{32}$. Weinberger and colleagues ${ }^{32}$ established a strong relationship between candiduria and use of wide spectrum antibiotics such as carbapeneme or cephtazidim in their series of 751 patients (for meropeneme $\mathrm{p}<0.001$, for cephtazidim $\mathrm{p}<0.001)$. Kobayashi et $a l^{28}$, reported that history of antibiotic use was present in $100 \%$ of the patients. Antimicrobial treatment was reported to be a risk factor for candiduria in $70-100 \%$ in various studies. It was found to be $45.5 \%$ in cases of this research $(\mathrm{p}=0.02)$. Steroids was also reported to be a risk factor for the development of candiduria ${ }^{33}$. Corticosteroids suppress normal immune mechanisms of the host against candida species. It was thought that the decrease in host immunity resulted in an increase in the virulence of fungi ${ }^{33}$. Orovcova and colleagues ${ }^{34}$ repoted that $72 \%$ of the patients with candiduria received corticosteroids treatment and they concluded that this treatment modality was among the risk factors of candidal infections. In the present study $31.8 \%$ received corticosteroids $(\mathrm{p}=0.2)$. Fungal infections are observed frequently in malignant subjects $^{34}$. It has been reported that besides the bacterial infections, which occur concomitantly with febrile neutropenic attacks, previously undetected Candida infections also manifested themselves in the form of candidemia and urinary tract infections ${ }^{35}$. In this study, $63.6 \%$ of patients with genitourinary tract cancer showed candiduria in comparison to $36.4 \%$ in other types of cancer $(p=0.06)$. Surgery is known be a risk factor for urinary Candidiasis; however, its mechanism is unclear. Ang and colleagues $^{22}$ showed that history of surgical intervention including the urinary system performed in the last two weeks was present in $73 \%$ of the patients with candiduria. Kobayashi and colleagues ${ }^{28}$ reported that there was history of surgical intervention in $66.7 \%$ of the patients with candiduria, and the presence of surgical intervention was established to be an 
effective risk factor for the development of candiduria. History of abdominal surgery was found in $13.6 \%$ of patients $(p=0.03)$. Candida albicans is the most common isolate in urine specimens ${ }^{32}$. Among all fungi isolated from the urine, $40-65 \%$ was found to be candida albicans ${ }^{35}$. Kauffman and colleagues ${ }^{27}$ reported that C. albicans was present in $51.8 \%$ and C. glabrata was present in $15.6 \%$ of the patients with candiduria, in their study performed with 751 patients. Weinberger and colleagues isolated C.albicans in $56.4 \%$, C. tropicalis in $19 \%$, C. glabrata in $15.7 \%$, C. parapsilosis is in $6.1 \%$ and C. krusei in $1.8 \%$ of the cases ${ }^{32}$. Although the prevalence of candida albicans is higher, the proportion of non- candida fungi involved in urinary pathogenesis increases over the course of time ${ }^{35}$. Orovcova and colleagues ${ }^{34}$ observed candida albicans in $72 \%$ of the cases and non- candida albicans pathogens in $28 \%$, in their series of 50 patients. The most common pathogen was candida albicans (21-72\%), and candida glabrata came the second (5-33\%). In this study, Candida albicans was determined in $35.7 \%$, candida tropicalis in $21.4 \%$, candida glabrata in $17.9 \%$, candida stellatoides in $14.3 \%$ of the cases and in $3.6 \%$ for each of candida guilliermondii, candida parapsilosis and candida krusei. Candida albicans was found to be the most common species and this finding was consistent with those of the other studies. Germ tube test method failed to discriminate between Candida albicans and Candida tropicalis as two isolates of the latter gave positive test. So, it is not a reliable test for identification of Candida albicans and this was consistent with Hazan and Howell ${ }^{2}$ and Badawi $^{24}$. C. tropicalis was absolutely identified on CMA but this media failed in discrimination of C. glabrata, that was in accordance with the finding of Koehler et al. ${ }^{1}$, Yticesoy and $\mathrm{Marol}^{9}$ and Badawia et al. ${ }^{24}, \mathrm{C}$. albicans, C. tropicalis and C. krusei were absolutely identified on CMA, but this media failed in discrimination of C. glabrata. Since C. glabrata on CMA is similar in appearance to other Candida species this explains failure of CMA for C. glabrata detection. Direct inoculation from primary plates of SDA providing the medium with a high concentration of species- specific enzyme gives higher accuracy for CMA than direct inoculation of clinical samples ${ }^{36}$. Sultan et al..$^{37}$, sated that further methods were needed for confirmation of identification of C. glabrata. The sensitivity to fluconazole in this study was only $42.86 \%$ using E-test method that was in agreement of $95.5 \%$ with broth microdilution method. E-test is an accurate method for MIC determination with numerous bacteria, including fastidious microorganisms and also Candida species $^{38}$. E-test on SDA media was preferred with Badawi et al. ${ }^{24}$, but not preferred by Tapia et $a l^{14}$, who preferred using RPMI 1640 for detection of antifungal resistance by E-test. Totorano et al. $^{39}$, revealed that interprettation of MICs by E test was more difficult in RPMI 1640 due to marked trailing endpoints observed in this medium with most of the strains caused by partial inhibition of fungal growth. E test is a simple, rapid, and easy for interpretation thus can be an alternative to NCCLs reference method (BM) for antifungal susceptibility. In conclusion; emergency of non Candida albicans species began to increase. Screening for fungal urinary tract infections is needed for high risk groups including cancer patients with accurate, simple identification methods to the species level with routine antifungal susceptibility testing. It is recommended to use API 20C AUX to detect the species level as it is accurate, simple and available. In addition, it is recommended to use E-test for determination of sensitivity of Candida species to antifungal agent as it is accurate and simple. Early detection of Candida urinary tract infections will prevent emergency of Candida resistant strains, decrease the complications of invasive candidiasis, the development of complicated urinary tract infections and improve the prognostic outcome.

\section{REFERENCES}

1- A. P. Koehler, K. C. Chu, E. T. S. Houang and E. T. S. Cheng, "Simple reliable cost effective yeast identification scheme for the clinical laboratory", J. Clin. Microbiol., 37 (2), 422-426 (1999).

2- K. C. Hazen and S. A. Howell, "Candida, Cryptococcus, and other yeasts of medical importance", In P. R. Murray, E. J. Baron, J. H. Jorgensen, M. A. Pfaller, and R. H. Yolken, (ed.), "Manual of Clinical Microbiology", 8th ed., ASM Press, Washington, D. C., 2003, pp. 1693-1711. 
3- O. Gudlaugsson, S. Gillespie, K. Lee and J. Vande Berg, "Attributable mortality of nosocomial candidemia revisited", Clin. Infect. Dis., 37, 1172-1177 (2003).

4- G. P. Moran, D. J. Sullivan and D. C. Coleman, "Emergence of non-Candida Albicans Species as Pathogens", In Candida and Candidiasis 1S' edition. Edited by Calderone RA. Washington D.C., American Society for Microbiology, 2003, pp. 37-53.

5- E. Karabatsaki, E. Kalogeropoulou and C. Kakari, "Characteristics of Patient Population with Funguria in a General Medical Department" $\quad 11^{\text {th }}$ European Congress of Clinical Microbiology and Infectious Diseases, Istanbul, Turkey, 2001, p. 337.

6- H. Erbay, A. N. Yalcin, S. Serin, H. Turgut, E. Tomatir, B. Cetin and M. Zencir, "Nosocomial infections in intensive care unit in a turkish university hospital, a 2-year survey", Intensive Care Med., 29, 1482-1488 (2003).

7- K. B. Laupland, D. A. Zygun, H. D. Davies, D. L. Church, T. J. Louie and C. J. Doig, "Incidence and risk factors for acquiring nosocomial urinary tract infection in the critically ill", I. Crit. Care, 17, 50-57 (2002).

8- Y. Morera, J. M. Torres-Rodriguez and I. Catalan, "Candiduria in patients with urethral catheter admitted in Intensive Care unit, etiology and in-vitro susceptibility to fluconazole", Med. Clin., (Bare)., 27, 118 (15), 580-2 (2002).

9- M. Yticesoy and S. Marol, "Performance of chrom agar candida and biggy agar for identification of yeast species", Annals of Clin. Microbiol. and Antimicrob., 2 (8), 1-7 (2003).

10- J. S. Heelan, E. Sotomayor, K. Coon and B. J. D'arezzo, "comparison of the rapid yeast plus panel with the api $20 \mathrm{c}$ yeast system for identification of clinically significant isolates of candida species", J. Clin. Microbiol., 36 (5), 14431445 (1998).

11- L. Buchaille, A. M. Freydiere, R. Guinet and Y. Gill, "Evaluation of six commercial systems for identification of medically important yeasts" Eur. J. Clin. Microbiol. Infect. Dis., 17, 479-488 (1999).

12- M. Ozalp, M. Ekizoglu and D. Gur, "InVitro Activity of Fluconazole and Amphotericin B Against Hospital Isolates of Candida species", European Congress of
Clinical Microbiology Infectious Diseases, Istanbul, Turkey, 2001, p. 343.

13- M. J. Matar, L. Ostrosky -Zeichner and V. L. Paetznick, "Correlation between e-test, disk diffusion and microdilution methods for antifungal susceptibility testing of fluconazole and voriconazole", Antimicrob. Agents Chemother., 47 (5), 1647-1651 (2003).

14- C. Tapia, E. Leon and E. Palavecino, "Antifungal susceptibility of yeasts by Etest comparison of 3 media", Rev. Med. Chil., 131 (3), 299-302 (2003).

15- N. E. Tolkoff-Rubin, R. S. Cortan and R. H. Rubin, "Urinary Tract Infection, Pyelonephritis, and Reflux Nephropathy, In: Brenner and Rector's the kidney", $6^{\text {th }}$ ed., Vol. 2, Ch. 31, 2000, pp. 1449-1508, Brenner, BM ed W.B Saunder Company, Philadelphia.

16- J. E. Hoppe and P. Frey, "Evaluation of six commercial tests and the germ tube test for presumptive identification of candida albicans", Eur. J. Clin. Microbiol. Infect. Dis., 18, 188-191 (1999).

17- A. M. Freydiere, R. Guinet and P. Boiron, "Yeast identification in the clinical microbiology laboratory: phenotypical methods", Medical Mycology., 39, 9-33 (2001).

18- G. Smith, An Introduction to Industrial Mycoloogy $5^{\text {th }}$ ed., Edward Arnold Ltd., London (1960).

19- M. A. Pfaller, B. M. Buschelman, M. Lancaster, A. Espinel-Ingroff, J. H. Rex, M. G. Rinaldi, C. R. Cooper, and M. R. McGinnis, "Quality control guidelines for national committee for clinical laboratory standards: recommended broth macrodilution testing of amphotericin b, fluconazole and flucytosine", J. Clin. Microbiol., 33, 1104-1107 (1995).

20- A. Colombo, F. Barchiesi, D. A. McGough, A. W. Fothergill, A. Bolmstrom and M. G. Rinaldi, "Antifungal Susceptibility Testing of Yeasts with Etest Azole Strips: A Comparison with the NCCLs Macrodilution Broth in the Evaluation of 80 Clinical Yeast Isolates", XII congress of the International Society for Human and Animal Mycology (1994).

21- A. W. Topcu and N. Cerikcioglu, Candida tiirleri., In: Topcu AW, SOyletir G, Doganay $\mathrm{M}$, eds. Infeksiyon hastahklan ve 
mikrobiyolojisi: Ankara: Nobel tip kitabevi, 2002, pp. 1797-1808.

22- B. S. P. Ang, A. Telenti, B. King, J. M. Steckelberg and W. R. Wilson. "Candidemia from a urinary tract source: microbiological aspects and clinical significance", Clin. Infect. Dis., 17, 662-666 (1993).

23- K. Pakshir, M. Moghadami, M. Emami and P. Kord Bacheh, "Prevalence and identification of etiological agents of funguria in Foley catheterized patients", J. Med. Res. Shiraz. Univ. Med. Sci., 3, 33-41 (2004).

24- H. Badawi, A. I. Kamel, N. Fam, M. El-Said and S. Elian, "Candida urinary infections: emerging species, antifungal susceptibility trends and antibody response", Egypt J. Med. Microbiol., 13 (1), 1-14 (2004).

25- N. Febre, V. Silva, E. A. S. Medeiros, S. B. Wey, A. L. Colombo and O. Fischman, "Microbiological characteristics of yeasts isolated from urinary tracts of intensive care unit patients undergoing urinary catheterization", J. Clin. Microbiol., 37, 1584-1586 (1999).

26- P. G. Pappas, J. H. Rex, J. D. Sobel, S. G. Filler,W. E. Dismukes, T. J. Walsh and J. E. Edwards, "Guidelines for treatment of Candidiasis", Clin. Infect. Dis., 38, 161-89 (2004).

27- C. A. Kauffman, J. A. Vazquez, J. D. Sobel, H. A. Gallis, D. S. McKinsey, A. W. Karchmer, A. M. Sugar, P. K. Sharkey, G. J. Wise, R. Mangi, A. Mosher, J. Y. Lee, and W. E. Dismukes, "Prospective multicenter surveillance study of funguria in hospitalized patients", ibid., 30, 14-18 (2000).

28- C. C. B. A. Kobayashi, F. L. Fernandes, K. C. Mirande, E. Souse and M. R. Silva, "Candiduria in hospital patients: A study prospective", Mycopathologia, 158, 49-52 (2004).

29- J. D. Sobel, C. A. Kauffman, D. McKinsey, M. Zervos, J. A. Vazquez, A. W. Karchmer, J. Lee, C. Thomas, H. Panzer and W. E. Dismukes, "Candiduria: A randomized, double-blind study of treatment with fluconazole and placebo", Clin. Infect. Dis., 30, 19-24 (2000).

30- L. G. Jacobs, E. A. Skidmore, L. A. Cardoso and F. Ziv., "Bladder irrigation with amphotericin B for treatment of fungal urinary tract infections", ibid., 18, 313-318 (1994).
31- A. D. Harris, J. Castro, D. C. Sheppard, Y. Carmeli and M. H. Samore, "Risk factors for nosocomial candiduria due to Candida glabrata and Candida albicans", ibid., 29, 926-928 (1999).

32- M. Weinberger, S. Sweet, L. Leibovici, S. D. Pitlik and Z. Samra, "Correlation between candiduria and departmental antibiotic use", J. Hosp. Infect., 53, 183-186 (2003).

33- T. Lundstrom and J. Sobel, "Nosocomial candiduria" a review, CID, 32, 1602-1607 (2001).

34- E. Orovcova, J. Lacka, L. Drgona, M. Studena, L. Sevcikova and S. Spanik, "Funguria in cancer patients: analysis of risk factors, clinical presentation and outcome in 50 patients", Infection, 24, 319-323 (1996).

35- J. L. Vincent, E. Anaissie, H. Bruining, W. Demajo, M. EI-Ebiary and J. Haber, "Epidemiology, diagnosis and treatment of systemic Candida infection in surgical patients under Intensive care", Intensive Care Med., 24, 206-216 (1998).

36- B. Willinger, C. Hillowoth, B. Selitsch and M. Manafi, "Performance of Candida ID, a new chromogenic medium for presumptive identification of Candida species, in comparison to CHROMagar Candida", J. Clin. Microbiol., 39, 3793-3795 (2001).

37- M. F. Sultan, A. F. Mostafa and M. F. Negm, "Rapid identification of yeast species from clinical samples using albicans ID and CHROM agar Candida plates", Egypt J. Med. Microbiol., 7 (34), 505-512 (1998).

38- A. Favel, C. Chastin, A. L. Thomet, P. Regli, A. Michel-Nguyen and A. Penaud, "Evaluation of the $\mathrm{E}$ test for antifungal susceptibility testing of Candida glabrata", Eur. J. Clin. Microbiol. Infect. Dis., 19, 146148 (2000).

39- A. M. Tortorano, M. A. Viviani, F. Barchiesi, "Comparison of three methods for testing azole susceptibilities of C. albicans strains isolated sequentially from oral cavities of AIDS patients", J. Clin. Microbiol., 36 (6), 1578-1583 (1998). 